\title{
First episode psychosis with extrapyramidal signs prior to antipsychotic drug treatment
}

\author{
HONER William $\mathrm{G}^{1 *}$, LANG Donna $\mathrm{J}^{2}$, KOPALA Lili $\mathrm{C}^{1,3}$, MACEWAN G William ${ }^{1}$, \\ SMITH Geoffrey $\mathrm{N}^{1}$, CHEN Eric $\mathrm{Y} \mathrm{H}^{4}$ \& CHAN Raymond $\mathrm{C} \mathrm{K}^{5}$ \\ ${ }^{1}$ Department of Psychiatry, University of British Columbia, Vancouver, BC, Canada; \\ ${ }^{2}$ Department of Radiology, University of British Columbia, Vancouver, BC, Canada; \\ ${ }^{3}$ Department of Psychiatry, Dalhousie University, Halifax, NS, Canada; \\ ${ }^{4}$ Department of Psychiatry, University of Hong Kong, Hong Kong, China; \\ ${ }^{5}$ Key Laboratory of Mental Health, Institute of Psychology, Chinese Academy of Sciences, Beijing 100101, China
}

Received March 19, 2011; accepted June 15, 2011; published online September 23, 2011

Extrapyramidal movement disorders are common in chronic schizophrenia, and may be an intrinsic feature of the illness as well as related to antipsychotic drug treatment. Similar dysfunctions at illness onset may have implications for outcome, and for understanding the mechanisms of illness. The objectives were to examine the clinical correlates of pre-treatment movement disorders at first episode of psychosis, and determine associations with neuropsychological function and striatal structure. Never medicated subjects were recruited from consecutive admissions to Early Psychosis Programs with defined catchment areas in Hong Kong, China, and Halifax, Canada. Standardized clinical, neuropsychological and brain imaging assessments were carried out at baseline and following acute and long term treatment with typical or atypical antipsychotic drugs. At the Hong Kong site, we studied 84 subjects with first episode psychosis ( $n=10$ with EPS). At the Halifax site, we studied 40 subjects with first episode psychosis ( $n$ $=17$ with EPS), and 23 healthy comparison subjects. Subjects with movement disorders prior to treatment (EPS+) had higher total PANSS scores at baseline (mean elevation 19.9\% Hong Kong, $P=0.016 ; 14.7 \%$ Halifax, $P=0.049$ ). In subjects treated with atypical antipsychotics (all Halifax), EPS+ status at baseline predicted more movement disorders at long term follow up $(P=$ 0.0005). In both cohorts, EPS+ subjects had poorer acute symptomatic treatment response assessed with the PANSS (Hong Kong $P=0.005$; Halifax $P=0.017$ ). Neuropsychological impairment related to executive dysfunction appeared greater in a small sample of EPS+ subjects (Hong Kong, effect size 0.26-0.27, $P<0.05$ ). Caudate volumes were $4.5 \%$ larger in EPS+ compared with EPS-subjects (Halifax $P=0.042$ ), and correlations between striatal volumes and age were different in the EPS+ group. In conclusion, pre-treatment EPS is present in a substantial minority of subjects with first episode psychosis, appears to persist at long term follow up, and is associated with poorer response of symptoms to treatment. Selective impairment of executive function and striatal enlargement provides evidence of abnormalities of brain function and structure associated with this aspect of early psychosis.

schizophrenia, movement disorder, basal ganglia, antipsychotics, cognitive, psychosis

Citation: Honer W G, Lang D J, Kopala L C, et al. First episode psychosis with extrapyramidal signs prior to antipsychotic drug treatment. Chinese Sci Bull, 2011, 56: 3361-3371, doi: 10.1007/s11434-011-4738-y

Signs of extrapyramidal dysfunction including akinesia, tremor, dystonia and dyskinesia were recognized in patients with schizophrenia prior to use of antipsychotic drug treatments [1-4]. Many of these same signs are described in family members of patients [5,6]. Most endophenotype studies

*Corresponding author (email: honer@interchange.ubc.ca) investigate neurological soft signs, that appear to represent signs of developmental deviance [7,8]. Extrapyramidal dysfunction may link more closely to the neuropharmacological features of schizophrenia, but must be differentiated from the effects of treatments used for the illness. With the introduction of antipsychotic drugs in the 1950s, and particularly with the development of high affinity dopamine D2 
antagonists, extrapyramidal signs became commonplace, complicating the investigation of movement disorders intrinsic to schizophrenia. However, implementation of early identification and intervention strategies for psychosis has reawakened interest in the movement disorders in schizophrenia, as has the search for endophenotypes of the illness.

Contemporary studies indicate acutely ill, never medicated patients may have signs of Parkinsonism (4\%-28\%), dystonia $(2 \%-17 \%)$ and dyskinesia $(1 \%-14 \%)$ [9-18]. Clinical correlates of extrapyramidal signs in first episode psychosis include increased negative symptoms with akinetic Parkinsonism in some $[9,17,18]$, but not all studies [15]. Lower education was a predisposing feature for dyskinesia prior to treatment [11]. Recent studies of first episode patients report that poor premorbid functioning and possibly a family history of severe mental illness were associated with signs of extrapyramidal dysfunction prior to antipsychotic exposure $[17,18]$.

Several lines of evidence, including a series of studies of older, chronic, never medicated patients suggest that movement disorders may be an integral part of schizophrenia $[19,20]$. Dyskinesia was the main manifestation in a chronic patient cohort, but Parkinsonism was also present $[21,22]$. Follow up studies over 18 months showed the movement disorders fluctuated over time, with $57 \%$ affected by dyskinesia and $35 \%$ affected by Parkinsonism on at least one assessment [22]. Dyskinesia was not related to memory dysfunction [23]. These patients showed enlargement of the left lentiform nucleus, and loss of the negative correlation between age and volumes of the caudate and lentiform nuclei [21].

Similarly detailed studies in early psychosis could be equally informative. The present investigation was designed to test the hypotheses that extrapyramidal signs at first presentation of psychosis are associated with clinically relevant features of illness, predict response of symptoms to treatment, and are associated with neuropsychological dysfunction and structural abnormalities of the striatum.

\section{Subjects and methods}

\subsection{Subjects}

Data was obtained from subjects treated by first episode psychosis programs in Hong Kong and in Halifax, and from healthy comparison subjects recruited in Halifax. All subjects provided written informed consent, and protocols were approved by the respective Ethics Committees. The Hong Kong sample was obtained from 153 consecutive first admissions for psychosis to Queen Mary and Pamela Youde Nethersole Eastern Hospitals, serving the Hong Kong Island catchment area (population 1300000). The time period was September, 1997 to March, 2000. The Halifax sample was obtained from approximately 250 consecutive referrals to the Nova Scotia Early Psychosis Program, which serves a
Provincial population of 940000 . The time period was December, 1995 to March, 2000.

Each program obtained standardized assessments of subjects at entry and after follow up. Assessments were made by program psychiatrists, trained in research methodology. Where possible, subjects were assessed prior to initiating antipsychotic treatment (Hong Kong $n=84$, Halifax $n=40$ ). The majority of subjects remained in treatment for at least 1 year. At both sites, inclusion criteria were: no previous antipsychotic drug treatment, a first episode psychotic disorder established according to DSM-IV criteria using all available clinical information after at least 6 months of assessment, age 13-65, in- or out-patients. Exclusion criteria were: known neurological disorder, or diagnosis of psychosis related to substance abuse. As part of the Hong Kong site protocol, subjects were administered neuropsychological tests. An additional inclusion criterion was fluency in Cantonese. Subjects with a history of special school attendance (usually indicating moderate to severe learning disability) were excluded. As part of the Halifax site protocol, subjects could participate in an MRI scan. Healthy comparison subjects were recruited from hospital staff and the local community. For the MRI study, exclusion criteria were a history of significant head injury or loss of consciousness greater than $5 \mathrm{~min}$, a history of DSM-IV substance abuse or seizure disorder, and a family history of psychosis (for comparison subjects).

\subsection{Assessment of extrapyramidal signs}

Assessments were made prior to antipsychotic treatment. For the Hong Kong sample, subjects were assessed with the Simpson-Angus Scale (SAS) [24], and the Abnormal Involuntary Movement Scale (AIMS) [25]. The SAS is comprised of 10 items, each rated $0-4$, subjects in the present study scored from $0-2$. A score of 0 indicates normal, 1 indicates a mild level of severity, 2 indicates moderate. The AIMS is comprised of 7 individual items rated $0-4$, subjects in the present study scored from 0-2. A score of 0 indicates normal, 1 indicates minimal severity, 2 indicates mild severity. Trained raters demonstrated acceptable inter-rater reliability in assessing movement disorders using a set of 15 videotaped examinations (intraclass correlation coefficient 0.93). Halifax subjects were assessed with the Extrapyramidal Syndrome Rating Scale (ESRS) [26]. The global scores are rated $0-8$, subjects in the present study scored from 0-2. A score of 0 indicates normal, 1 indicates borderline and 2 indicates a definite but very mild level of severity. Inter-rater reliability for the ESRS was good (intraclass correlation coefficient 0.84 ).

The approaches to assessing EPS at the two sites involved the use of different, but standardized and widely accepted scales. Compared with the SAS, the ESRS contains more items assessing a variety of features of Parkinsonism and also assesses dystonia. The use of different 
scales precludes pooling data between sites. However, convergent evidence obtained with different strategies also has advantages. The individual items of the SAS and AIMS, and the subscales of the ESRS assess multiple domains of extrapyramidal dysfunction. Following antipsychotic drug treatment, there is generally a significant inter-correlation between scores on individual items or subscales, and summary scores can be used for analysis. However, in never medicated subjects with schizophrenia there may be no inter-correlation between individual items or subscales [17,27], and summary scores may fail to detect subtle but definite signs of extrapyramidal dysfunction. We categorized subjects according to the presence or absence of definite signs of extrapyramidal dysfunction. Scores of 1 or more on individual items of the SAS or AIMS, and scores of 2 or more on the global subscales of the ESRS were used for this purpose.

\subsection{Assessment of symptoms and treatment response}

Symptom severity prior to antipsychotic drug treatment was rated using the Positive and Negative Syndrome Scale (PANSS) [28]. This is a 30 item scale, with items rated 1-7. There are three subscales, for positive and negative symptoms (7 items each), and for general psychopathology (16 items). In the Hong Kong sample, PANSS scores were available for 80/84 subjects for the acute treatment response analysis. All were treated with typical antipsychotic medications (mean $358 \mathrm{mg} \mathrm{CPZ} \mathrm{equivalents,} \mathrm{SD} \mathrm{461)} \mathrm{for} \mathrm{a} \mathrm{mean}$ of 6.2 weeks (SD 4.1). PANSS scores were available for $57 / 84$ subjects for the long term treatment response analysis (mean $254 \mathrm{mg}$ CPZ equivalents, SD 252). At both time points the mean doses in subjects with and without pre-existing EPS did not differ. In the Halifax sample, acute treatment response PANSS scores were available for 28/40 subjects. All were treated with atypical antipsychotic medications (risperidone $n=17$, mean dose $2.9 \mathrm{mg}$, olanzapine $n$ $=4$, mean dose $16.9 \mathrm{mg}$, quetiapine $n=7$, mean dose 400 $\mathrm{mg}$ ) for a mean of 10.3 weeks (SD 2.5). Long term treatment response data was available for $26 / 40$ subjects. All were treated with atypical antipsychotic medications (risperidone $n=12$, mean dose $2.7 \mathrm{mg}$, olanzapine $n=7$, mean dose $13.6 \mathrm{mg}$, quetiapine $n=7$, mean dose $393 \mathrm{mg}$ ) for a mean of 45.6 weeks (SD 15.8). For both cohorts, the duration of treatment for subjects with baseline EPS did not differ from the no EPS group, and medication dosages were similar between groups.

Inter-rater reliability for the PANSS was assessed at each site and found to be acceptable (intraclass correlation coefficients 0.83 Hong Kong, 0.85 Halifax).

\subsection{Cognitive testing}

A battery of cognitive tests was used as part of the Hong Kong protocol. Testing was carried out as soon as feasible following admission to hospital, and all subjects in the pre- sent report were tested prior to receiving any medication. For the 10 subjects with EPS at baseline, we selected 10 comparison subjects from the group with no EPS. The basis of selection was the intent to match the two groups as closely as possible for age, education, gender, and duration of untreated psychosis.

An index of overall premorbid cognitive function was assessed with the information subscale of the Wechsler Adult Intelligence Scale (WAIS-R-HK, Revised Cantonese Version, Hong Kong Psychological Society, 1989). Scaled scores were used in analysis. Memory was assessed with the logical memory and visual reproduction subscales of the Wechsler Memory Scale. In the Logical Memory Test [29] (adapted for Cantonese-speaking patients, Wong C. W., personal communication), subjects were requested to memorise a verbally presented story, then repeat the story immediately and after a 30-min delay. In the Visual Reproduction Test, subjects were instructed to memorize a visually presented design for $10 \mathrm{~s}$, then to draw the design from memory immediately afterwards and following a 30-min delay. Executive functions were assessed with verbal fluency and the Modified Wisconsin Card Sorting Test (MWCST)[30] ( $n=8$ for this test). In the verbal fluency test, subjects were required to name as many exemplars as possible from the category "animal" within $1 \mathrm{~min}$. In the MWCST, subjects were asked to sort a set of 48 cards according to three sorting principles (color, shape and number). Subjects were instructed that the sorting principle would change after they made 6 consecutive correct responses. The numbers of both preservative and total errors were monitored.

\subsection{Magnetic resonance imaging}

Imaging was carried out as part of the Halifax protocol. Patients were scanned as soon as possible following initial assessment. MRI scans were carried out using a Siemens Magnaton Vision 1.5T scanner. The inversion recovery sequence $(\mathrm{TR} / \mathrm{TE}=2000 / 20 \mathrm{~ms}, \mathrm{FOV}=200 \mathrm{~cm}$, matrix $168 \times 256)$ generated 18 coronal slices $4 \mathrm{~mm}$ thick with $1 \mathrm{~mm}$ gaps. The gray-white contrast ratio was 1.42 , better than a spoiled gradient recall acquisition sequence (0.89). Five slices spanned the basal ganglia. A single operator outlined the caudate, putamen and globus pallidus with a previously described protocol $[31,32]$. Intraclass correlation coefficients for inter-rater reliability with this protocol were: caudate 0.99 ; putamen 0.97 , globus pallidus 0.96 , intracranial volume 0.99 . MRI scans were available at baseline for 33 patients, 32 of whom participated in a previously reported study [31].

\subsection{Statistical analysis}

(i) Baseline clinical variables. Comparisons between groups with and without EPS prior to treatment used $t$-tests for PANSS scores. Duration of untreated psychosis was not 
normally distributed, so a Mann-Whitney $U$-test was used.

(ii) Course of EPS during treatment. The frequencies of patients with and without EPS at baseline, and after acute and long term treatment were calculated using the definitions described above. Frequencies at each of these time points were compared with baseline frequencies using the Chi-square test.

(iii) Symptom response during treatment. Repeated measures analysis of variance was used, with main effects group (baseline EPS+, EPS-), time (baseline, outcome), and a group-by-time interaction term, with total PANSS score as the dependent measure. Due to loss of subjects with time, separate repeated measures analyses were made for acute and long term outcome time points.

(iv) Cognitive function. The adequacy of matching between the EPS+ and EPS- groups that received cognitive testing was assessed using $t$-tests to compare age, education, and duration of hospitalization between groups. Duration of untreated psychosis was compared with a Mann-Whitney $U$-test. Comparisons of cognitive performance between groups were made with the Mann-Whitney $U$-test.

(v) Basal ganglia structure. An analysis of covariance was used to compare volumes, with main effects group and gender, covariates intracranial volume and age, and a groupby-time interaction term [33]. Pearson correlation coefficients were calculated to investigate the relationship between age and volume of each structure, in each group (baseline EPS+, EPS-, control). A secondary analysis was carried out using a similar strategy as applied to investigate striatal structure in chronic, never mediated patients [21]. Three groups of pairs of subjects were formed ( $n=13$ /group), matched for gender and as closely as possible for age. Pairs were: EPS+ and EPS-, EPS+ and controls, EPS- and controls. Paired $t$-tests were carried out, as reported in the study of chronic subjects.

\section{Results}

\subsection{EPS in subjects with no previous antipsychotic treatment}

Demographic and clinical features of illness appear in Table 1. The overall prevalence of EPS was 10/84 (11.9\%) in the Hong Kong sample and 17/40 (42.5\%) in the Halifax sample. The predominant expression was Parkinsonism (see Table 2).

In the Hong Kong sample, EPS+ subjects had a longer duration of untreated psychosis (DUP, Mann-Whitney $U$-test, $Z=2.18, P=0.03$ ). The median DUP was also longer in the Halifax EPS+ subjects, but the difference was not statistically significant. At baseline, EPS+ subjects had higher total PANSS scores in both the Hong Kong (19.9\%, $t=2.45, P=0.016)$ and Halifax $(14.7 \%, t=2.04, P=0.049)$ groups (Figure 1). The Hong Kong EPS+ group had more severe positive $(t=2.00, P=0.049)$ and negative $(t=3.00$, $P=0.004)$ symptoms than the EPS- group. Differences

Table 1 Demographic and clinical variables for never medicated subjects with psychosis and healthy comparison subjects ${ }^{\text {a) }}$

\begin{tabular}{|c|c|c|c|c|c|}
\hline \multirow{2}{*}{ Variable } & \multicolumn{2}{|c|}{ Hong Kong } & \multicolumn{2}{|c|}{ Halifax } & \multirow{2}{*}{$\begin{array}{c}\text { Controls } \\
\text { (mean, SD) }\end{array}$} \\
\hline & EPS+ (mean, SD) & EPS- (mean, SD) & EPS+ (mean, SD) & EPS- (mean, SD) & \\
\hline$n$ (males/females) & $10(6 \mathrm{~m} / 4 \mathrm{f})$ & $74(35 \mathrm{~m} / 39 \mathrm{f})$ & $17(14 \mathrm{~m} / 3 \mathrm{f})$ & $23(17 \mathrm{~m} / 6 \mathrm{f})$ & $23(12 \mathrm{~m} / 11 \mathrm{f})$ \\
\hline Education (year) & $10.1(2.0)$ & $10.6(3.4)$ & $11.4(1.7)$ & $11.8(1.7)$ & $15.8(4.6)$ \\
\hline Duration of untreated psychosis (d) & 730 (median)* $^{*}$ & $120($ median $)$ & 309 (median, $n=13$ ) & 230 (median, $n=16$ ) & \\
\hline \multicolumn{6}{|l|}{ Ethnicity $(n)$} \\
\hline Chinese & 10 & 74 & & & \\
\hline Caucasian & & & 16 & 19 & 22 \\
\hline African-American & & & 1 & 2 & \\
\hline First Nations & & & & 1 & \\
\hline Asian & & & & & 1 \\
\hline Other & & & & 1 & \\
\hline Schizophrenia & 8 & 37 & 16 & 21 & \\
\hline Schizoaffective & & & 1 & 1 & \\
\hline Schizophreniform & 1 & 15 & & 1 & \\
\hline Brief psychosis & & 8 & & & \\
\hline Delusional disorder & 1 & 10 & & & \\
\hline Depression with psychosis & & 2 & & & \\
\hline PNOS & & 2 & & & \\
\hline
\end{tabular}

a) Significant differences between EPS+ and EPS- groups are indicated $* P<0.05$. 
Table 2 Course of EPS with treatment ${ }^{\text {a) }}$

\begin{tabular}{|c|c|c|c|c|c|c|c|}
\hline & \multirow{3}{*}{ Baseline status } & \multicolumn{6}{|c|}{ Prevalence of EPS } \\
\hline & & \multicolumn{3}{|c|}{ Hong Kong } & \multicolumn{3}{|c|}{ Halifax } \\
\hline & & Baseline & 6 weeks & 26 weeks & Baseline & 10 weeks & 46 weeks \\
\hline \multirow{6}{*}{$\begin{array}{l}\text { Parkinsonism } \\
n(\%)\end{array}$} & EPS+ & $10 / 10$ & $6 / 9$ & $2 / 8$ & $14 / 17$ & $1 / 9$ & $6 / 11$ \\
\hline & & $(100)$ & $(66.7)$ & $(25)$ & $(82.4)$ & $(11.1)$ & $(54.5)$ \\
\hline & EPS- & $0 / 74$ & $25 / 73$ & $17 / 50$ & $0 / 23$ & $2 / 14$ & $1 / 15$ \\
\hline & & $(0)$ & $(34.2)$ & $(34.0)$ & $(0)$ & (14.3) & (6.7) \\
\hline & Total & $10 / 84$ & $31 / 82$ & $19 / 58$ & $14 / 40$ & $3 / 23$ & $7 / 26$ \\
\hline & & (11.9) & $(37.8)$ & $(32.8)$ & $(35.0)$ & (13.0) & (26.9) \\
\hline \multirow{6}{*}{$\begin{array}{l}\text { Dyskinesia } \\
n(\%)\end{array}$} & EPS+ & $1 / 10$ & $1 / 9$ & $0 / 8$ & $4 / 17$ & $0 / 9$ & $4 / 11$ \\
\hline & & $(10.0)$ & $(11.1)$ & $(0)$ & $(23.5)$ & $(0)$ & $(36.4)$ \\
\hline & EPS- & $0 / 74$ & $3 / 73$ & $2 / 50$ & $0 / 23$ & $0 / 14$ & $0 / 15$ \\
\hline & & $(0)$ & (4.1) & $(4.0)$ & $(0)$ & $(0)$ & $(0)$ \\
\hline & Total & $1 / 84$ & $4 / 82$ & $2 / 58$ & $4 / 40$ & $0 / 23$ & $4 / 26$ \\
\hline & & $(1.2)$ & $(4.9)$ & $(3.4)$ & $(10.0)$ & $(0)$ & $(15.4)$ \\
\hline \multirow{6}{*}{$\begin{array}{l}\text { Dystonia } \\
n(\%)\end{array}$} & EPS+ & & & & $6 / 17$ & $2 / 9$ & $3 / 11$ \\
\hline & & & & & (35.3) & $(22.2)$ & (27.3) \\
\hline & EPS- & & & & $0 / 23$ & $0 / 14$ & $0 / 15$ \\
\hline & & & & & $(0)$ & $(0)$ & $(0)$ \\
\hline & Total & & & & $6 / 40$ & $2 / 23$ & $3 / 26$ \\
\hline & & & & & $(15.0)$ & $(8.7)$ & (11.5) \\
\hline
\end{tabular}

a) For this table, patients were split into two groups according to baseline status prior to treatment: those with (EPS+) and those without (EPS-) extrapyramidal symptoms. These group assignments were maintained, and frequencies (percents) of EPS after acute and chronic treatment were tabulated. Typical antipsychotic drugs were used in the Hong Kong sample, atypical antipsychotics in the Halifax sample.
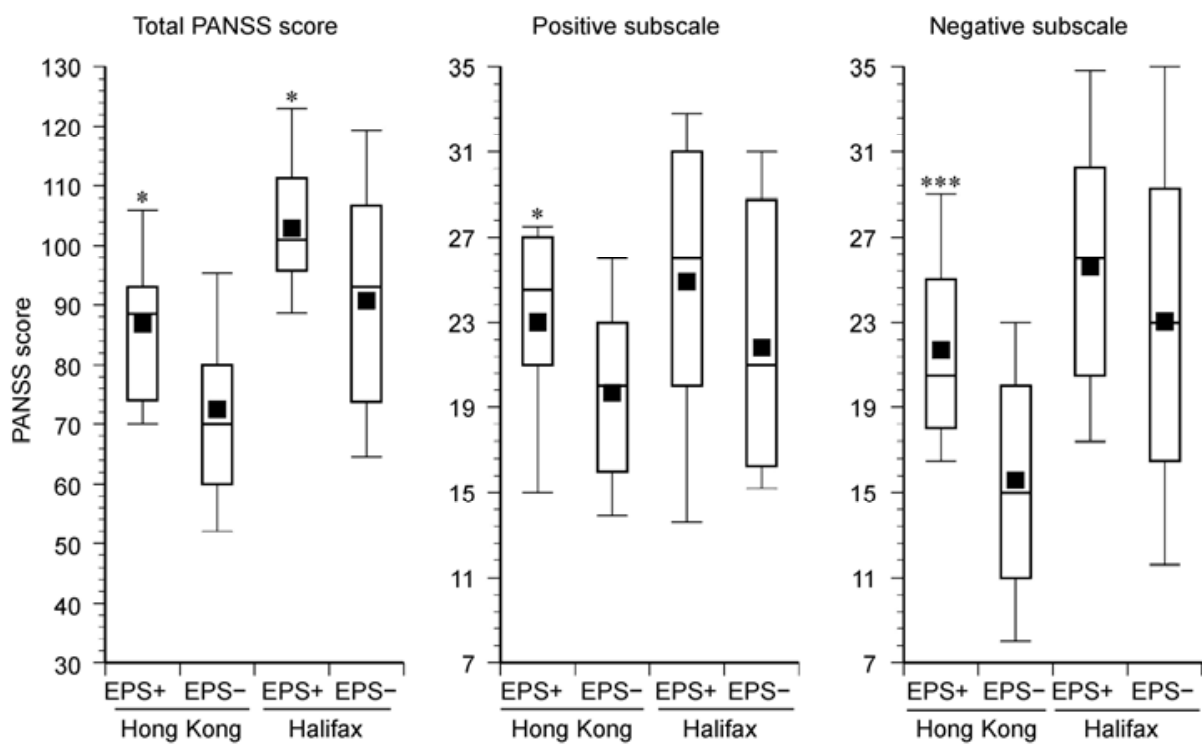

Figure 1 Severity of symptoms at baseline for never medicated subjects with psychosis. Boxplots indicate mean (square), and 95th, 75th, 50th, 25th and 5th percentiles. Significant differences between EPS+ and EPS- groups are indicated $* P<0.05, * * * P \leqslant 0.005$.

between groups were similar in direction in the Halifax cohort, but were not statistically significant.

\subsection{Course of EPS during treatment}

Treatment was with typical antipsychotics in the Hong Kong sample. In the overall group, EPS increased in frequency following a mean of 6 weeks of treatment, and remained elevated following a mean of 26 weeks of treatment
(Table 2, Figure 2). Dyskinesia developed in 2 subjects by the long term outcome point, both of whom were free of EPS at baseline. The likelihood of having EPS at either outcome time point was not related to EPS status at baseline (acute outcome Chi square $=3.25, P=0.07$; long term outcome Chi square $=0.37, P=0.57)$.

Treatment was with atypical antipsychotics in the Halifax sample. In the overall group, EPS declined in frequency after a mean of 10 weeks of treatment, but increased again 


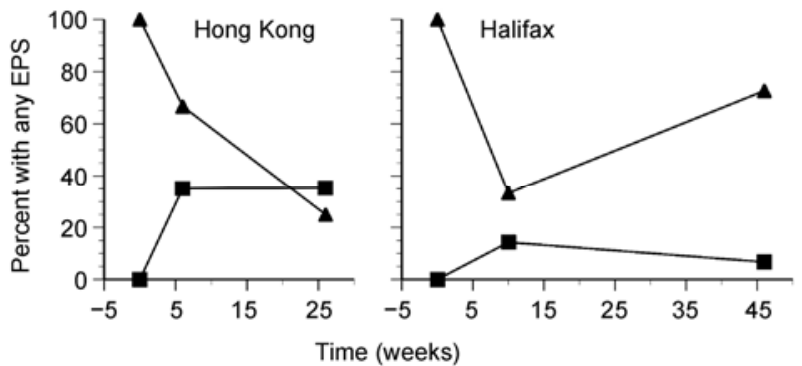

Figure 2 Change in prevalence of any signs of EPS over time in patient groups EPS+ prior to treatment (triangles) and in EPS- prior to treatment (squares). Subjects in the Hong Kong group were treated with typical antipsychotics, in the Halifax group atypical antipsychotics were used. The presence of EPS prior to treatment was related to persistent EPS in the Halifax group but not the Hong Kong group.

after a mean of 46 weeks of treatment. Dyskinesia was present in 4/26 (15.4\%) at long term follow up. Prior to antipsychotic treatment, one of these subjects had dyskinesia, three had evidence of Parkinsonism, and two had evidence of dystonia. None of the subjects who were free of EPS at baseline developed dyskinesia. At the acute treatment response time point, the frequency of EPS was not related to EPS status at baseline (Chi square $=1.17, P=0.28$ ). However, the likelihood of having EPS at long term outcome was related to EPS status at baseline (Chi square $=12.24, P$ $=0.0005)$. Subjects with pre-existing EPS were more likely to have EPS at long term follow up.

\subsection{Baseline EPS and symptomatic treatment response}

The patterns of response of symptoms are illustrated in Figure 3 . In both samples, repeated measures analysis of variance indicated statistically significant differences between EPS+ and EPS- groups following acute treatment (Hong Kong $F=8.58, P=0.005$; Halifax $F=6.53, P=0.017$ ). Patients with EPS at baseline had higher levels of total symptoms overall. This difference continued to be statisti- cally significant at the 26 week time point in the Hong Kong sample $(F=4.70, P=0.03)$ but was not significant in the Halifax sample at 46 weeks. There were no significant interactions between group and time. Concerning the acute response of positive symptoms, in both samples there were statistically significant differences related to the presence of baseline EPS (Hong Kong $F=4.26, P=0.043$; Halifax $F=$ $4.71, P=0.039)$. There were no statistically significant differences when the long term treatment response was considered, and there were no group-by-time interactions. Analysis of the acute treatment response of negative symptoms indicated effects related to presence of baseline EPS (Hong Kong $F=7.47, P=0.008$; Halifax $F=3.18, P=$ $0.09)$. These were persistent in the Hong Kong group only when the long term treatment response was considered $(F=$ $6.36, P=0.01)$. There were no group-by-time interactions in either sample.

\subsection{Baseline EPS and cognitive function}

Cognitive function was compared between demographically similar subgroups in the Hong Kong sample (Table 3). There were no differences in the index measure of premorbid global cognitive function. Two differences emerged on more specific tests, with relatively impaired performance in the EPS+ group. These were delayed recall in the visual reproduction test (approximate effect size 0.26) and increased perseverative errors in the WCST (approximate effect size 0.27). These results must however be treated with caution as the sample sizes were small and the results not corrected for multiple testing.

\subsection{Baseline EPS and basal ganglia structure}

At the time of MRI, 12 subjects were never medicated (4/13 with EPS, 8/20 without EPS). The mean duration of treatment for the remaining 21 subjects was 6.5 weeks (SD 3.5,
PANSS total

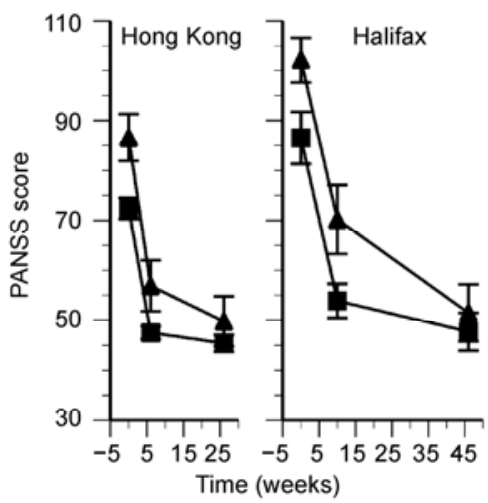

PANSS positive

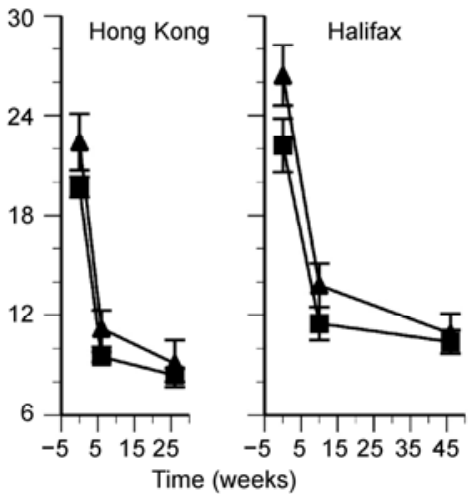

PANSS negative

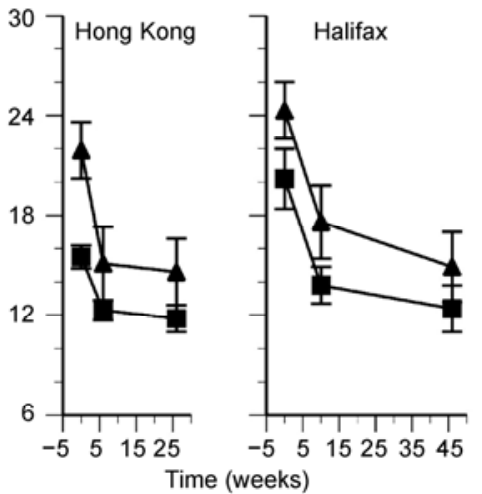

Figure 3 Change in symptoms over time in patient groups EPS+ prior to treatment (triangles) and in EPS- prior to treatment (squares). Values are mean and standard error of the mean. 
Table 3 Cognitive performance during first hospitalization in patients with EPS compared with a matched group with no EPS prior to antipsychotic drug treatment $^{\mathrm{a})}$

\begin{tabular}{|c|c|c|c|c|c|c|}
\hline & \multicolumn{2}{|c|}{ EPS+ } & \multicolumn{2}{|c|}{ EPS- } & \multirow{2}{*}{$t$-value/ $U$-value } & \multirow{2}{*}{$P$-value } \\
\hline & Mean/median & SD/range & Mean/median & SD/range & & \\
\hline \multicolumn{7}{|l|}{ Demographics } \\
\hline Age (year) & 28.10 & 6.38 & 28.50 & 6.29 & 0.14 & 0.89 \\
\hline Education (year) & 10.11 & 1.96 & 10.00 & 1.76 & 0.13 & 0.90 \\
\hline Gender (M/F) & $6 / 4$ & & $6 / 4$ & & & \\
\hline DUP (d) & 730 & $60-3285$ & 683 & $150-3650$ & 32.5 & 0.52 \\
\hline Duration of hospitalization (d) & 51 & 37 & 37 & 29 & 1.01 & 0.33 \\
\hline \multicolumn{7}{|l|}{ General cognition } \\
\hline Information subscale & 11.00 & $4-21$ & 11.00 & $7-21$ & 45 & 0.739 \\
\hline \multicolumn{7}{|l|}{ Memory } \\
\hline Logical memory immediate recall (\%) & 36.96 & $13.04-65.22$ & 26.09 & $13.04-39.13$ & 30 & 0.143 \\
\hline Logical memory delayed recall (\%) & 23.92 & $0-68.87$ & 15.22 & $4.35-43.48$ & 42 & 0.579 \\
\hline Visual reproduction immediate recall (\%) & 19.00 & $8-24$ & 20.00 & $10-25$ & 40 & 0.481 \\
\hline Visual reproduction delayed recall $(\%)^{*}$ & 17.00 & $0-22$ & 19.50 & $10-24$ & 22.5 & 0.036 \\
\hline \multicolumn{7}{|l|}{ Executive functions } \\
\hline Verbal fluency & 19.00 & $6-30$ & 17.50 & $10-28$ & 48 & 0.912 \\
\hline WCST perseverative error (number) ${ }^{*}$ & 10.00 & $4-47$ & 4.50 & $0-20$ & 12 & 0.012 \\
\hline WCST category & 3.50 & $0-4$ & 3.50 & $0-6$ & 30.5 & 0.408 \\
\hline
\end{tabular}

a) $* P<0.05$.

range 1.9-12.4), and did not differ between EPS+ and EPSsubjects. At the time of scan, 16 subjects were receiving risperidone (mean $2.9 \mathrm{mg}$ ), 2 received olanzapine $(15 \mathrm{mg}$ ), 1 received quetiapine $(300 \mathrm{mg}), 1$ received haloperidol (EPS-, 6 weeks, $3 \mathrm{mg}$ ) and 1 received a study drug (EPS-, 9 weeks, either risperidone or haloperidol).

Basal ganglia volumes are illustrated in Figure 4. Comparison of caudate volumes revealed a significant effect of group $(F=3.38, P=0.042)$. Volumes were larger in the EPS+ group (4.5\% vs. EPS-, 3.6\% vs. healthy subjects). No statistically significant differences were observed for putamen or globus pallidus volumes. The correlations between

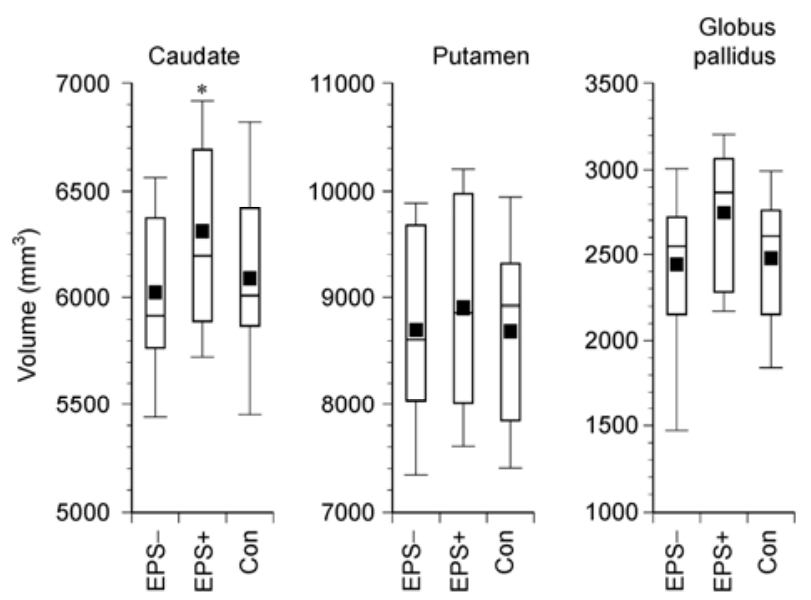

Figure 4 Basal ganglia volumes from MRI scans at baseline. Boxplots indicate mean (square), and 95th, 75th, 50th, 25th and 5th percentiles. $* P<0.05$. volume and age appear in Table 4. Caudate volume was negatively correlated with age in the EPS + group only $(P=$ $0.017)$. Putamen volume was negatively correlated with age in the healthy comparison group $(P=0.0006)$, and globus pallidus volume was also negatively correlated with age in this group $(P=0.012)$. Correlations between putamen or globus pallidus volumes and age were not statistically significant in either EPS group.

In subjects who participated in the MRI scan, 14/20 EPS- had a lifetime history of exposure to marijuana, versus 4/13 EPS+ (Chi-square $=4.89, P=0.027)$. Overall, when marijuana exposure was substituted for EPS in the model, subjects with marijuana exposure did not show a difference in caudate, putamen or globus pallidus volumes compared with those who had no exposure. In subjects who participated in the MRI scan, 1/20 EPS- had a lifetime history of previous exposure to stimulant drugs, versus $1 / 13$ EPS+. When these two subjects were excluded from the overall analysis, the statistical significance of the results was unchanged.

The paired analysis was carried out to replicate the strategy used to investigate volume differences between chronic, never medicated patients with and without movement disorders [21]. The results were similar to the analysis above for the caudate, and additional findings emerged for the putamen and the globus pallidus (Figure 5). For the caudate, volumes of the EPS+ group were $6.8 \%$ larger than the EPSgroup (paired $t=2.22, P=0.047$ ). For the putamen, the EPS- group volumes were $4.4 \%$ smaller than healthy comparison subjects (paired $t=2.39, P=0.033$ ). For the globus 
Table 4 Correlations between age and volumes of basal ganglia structures ${ }^{\text {a) }}$

\begin{tabular}{|c|c|c|c|c|c|c|c|}
\hline & \multirow[b]{2}{*}{$n$} & \multicolumn{2}{|c|}{ Caudate } & \multicolumn{2}{|c|}{ Putamen } & \multicolumn{2}{|c|}{ Globus pallidus } \\
\hline & & $r$ & $95 \%$ CI & $r$ & $95 \%$ CI & $r$ & $95 \% \mathrm{CI}$ \\
\hline EPS+ & 13 & $-0.64^{*}$ & $-0.88,-0.14$ & -0.52 & $-0.83,0.05$ & -0.11 & $-0.62,0.47$ \\
\hline EPS- & 20 & 0.07 & $-0.39,0.49$ & -0.20 & $-0.59,0.27$ & -0.08 & $-0.50,0.38$ \\
\hline Controls & 23 & -0.18 & $-0.55,0.25$ & $-0.64^{*}$ & $-0.84,-0.32$ & $-0.51^{*}$ & $-0.76,-0.12$ \\
\hline
\end{tabular}

a) These were statistically significant $* P<0.05$ for caudate in the EPS + group, and for putamen and globus pallidus in the control group.
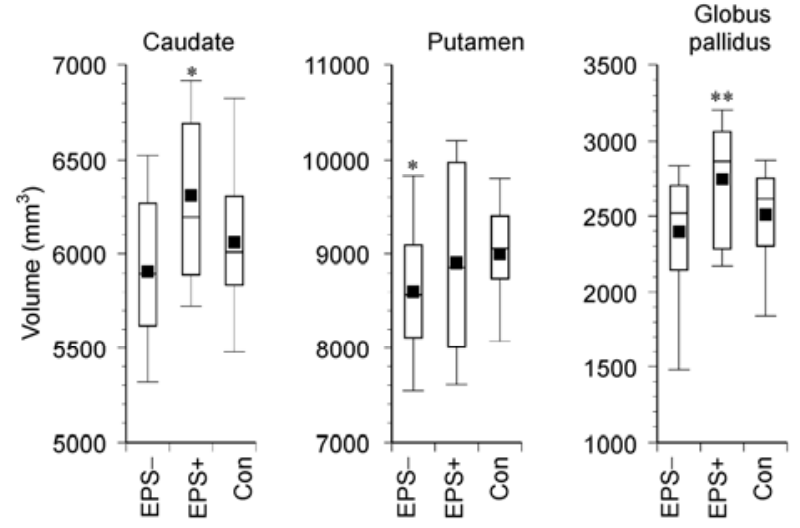

Figure 5 Basal ganglia volumes from MRI scans at baseline in groups matched for gender and age. Boxplots indicate mean (square), and 95th, 75th, 50th, 25th and 5th percentiles. $* P<0.05$, $* * P<0.005$.

pallidus, the EPS+ group volumes were $14.7 \%$ larger than EPS- group (paired $t=4.08, P=0.0015$ ). Both left and right volumes were similarly affected.

\section{Discussion}

The present results linking pre-treatment EPS to symptom severity in first episode psychosis, to cognitive dysfunction, and to abnormalities of brain structure all support the proposal that similar observations in family members of patients with psychosis represent an endophenotype of the illness [5,6]. Findings concerning the course of extrapyramidal symptoms and the relationships to treatment response indicate the possible importance of thorough assessment for EPS at the entry into early psychosis treatment programs.

The prevalence of EPS was within the range reported in early psychosis $[9-18,34,35]$. We used the presence of any EPS to form a subgroup for analysis. While subgrouping according to type of EPS would have been preferable, the sample size was not large enough, and Parkinsonism and dyskinesia may overlap significantly in never medicated subjects $[18,20,22]$. In both cohorts, higher total symptom severity scores were associated with pre-treatment EPS. In the Hong Kong cohort, both positive and negative symptom scores were also greater in the EPS+ group. These relationships are similar to those reported in the literature for total [17], for positive [34], and for negative symptoms [9,12,
16-18,34]. In chronic never-medicated subjects, these relationships were not observed [20]. One of our cohorts demonstrated a relationship between severity of EPS and duration of untreated psychosis, not seen elsewhere [16,17].

Risk of treatment-related Parkinsonism was unrelated to pre-existing EPS in the Hong Kong sample, where the acute treatment dose of typical antipsychotics was approximately $360 \mathrm{mg}$ CPZ equivalents/d. In contrast, pre-treatment EPS increased the risk for Parkinsonism in the acute phase of treatment with $20-40 \mathrm{mg} / \mathrm{d}$ of fluphenazine or haloperidol (approximately 1000-2000 CPZ equivalents) [16]. We did not find an association between pre-treatment EPS and risk for developing tardive dyskinesia related to typical antipsychotic drugs. A similar observation was made in the Hillside Hospital study [16,36]. In the Hong Kong cohort, baseline EPS appeared to decline over the first 6 months of typical antipsychotic treatment. In other studies, similar observations were reported for neurological soft signs [37,38].

Subjects in the Halifax cohort were treated with atypical antipsychotics. Subjects with EPS prior to treatment had reduced EPS after 10 weeks of treatment, similar to a report of 2-14 weeks of treatment with risperidone in subjects with baseline EPS [39]. A longer term study of subjects with baseline EPS indicated no change in overall severity after 6 months of treatment, largely with atypical antipsychotic drugs [34]. In the present study, later re-emergence of EPS in predisposed subjects after a mean of 46 weeks may represent progression of the movement disorder process, or development of tolerance to a suppressive effect of medications. As well, the initial dopamine receptor occupancy may eventually result in receptor up regulation which differs with different types of antipsychotic drugs [40].

The treatment response of psychopathology was less complete in EPS+ subjects in both cohorts in the present study. These results are similar to two other reports, where patients with baseline EPS present were less likely to respond to treatment, and when they did respond, this took longer [16,18]. However, these observations are in contrast to another study, where Parkinsonism at baseline appeared to predict extent of response of positive symptoms [34].

We observed poorer executive function in subjects with baseline EPS. There are few other studies of cognitive function in subjects with EPS prior to treatment. Older, never medicated subjects with dyskinesia appeared to have similar memory dysfunction on the Wechsler Memory Scale as those with no dyskinesia [23]. Global cognitive impairment 
was reported in three elderly, never medicated subjects [41]. In subjects with dyskinesia who were previously medicated with antipsychotic drugs, abnormalities of executive function were described $[42,43]$. Frontal-striatal-thalamic circuits involving the dorsolateral frontal cortex were implicated in this association [42]. Of note, prefrontal cortical dysfunction may contribute to presynaptic dopamine over activity in the striatum [44], or specifically the ventral striatum [45], an effect which is further modulated by COMT genotype [46].

This is the first MRI study separating groups of first episode patients according to the presence or absence of pre-treatment EPS. Some studies of the basal ganglia in first episode psychosis show no differences in volume from healthy subjects $[31,33,47-53]$, and others report smaller volumes [54-56]. One report of adolescent offspring of parents with schizophrenia demonstrated smaller caudate volumes in the at-risk probands [57]. The present findings of larger caudate nuclei and possibly larger globus pallidus, as well as disrupted correlations between age and basal ganglia volumes associated with pre-treatment EPS are consistent with a study of chronic, never medicated subjects [21]. In the chronic subjects, the left lentiform nucleus was enlarged in those with EPS compared with controls [21]. The predominant form of EPS was dyskinesia, however elevated scores for Parkinsonism were also observed. Relatively larger caudate nuclei were also reported in subjects with chromosome $22 q$ deletion syndrome, a significant genetic risk factor for schizophrenia [58-60].

The mechanism of volume enlargement is unknown, although several studies may provide clues. Dopamine depleting agents including cocaine and methamphetamine also appear to be associated with basal ganglia enlargement [61-64]. Typical antipsychotic drug treatment enlarges basal ganglia volume $[55,65,66]$, and these changes can be reversed by switching to atypical antipsychotics including clozapine and olanzapine [32,55,65-68]. Striatal dopamine synthesis in never medicated subjects with schizophrenia was generally reported to be increased [69-71]. However, individual, never medicated subjects with catatonia in several studies were reported to have lower dopamine synthesis than healthy subjects $[69,70]$. Treatment with haloperidol reduced dopamine synthesis in striatum and in thalamus, with associated increase in EPS [72]. Another study of medicated subjects indicated dorsal striatal regions may show relatively normal dopamine synthesis, while ventral regions show increased synthesis [45]. In this study, although regional volumes were not significantly different from healthy comparison subjects, the mean dorsal striatal volumes were larger than controls, while mean ventral striatal volumes were smaller. The possibility of regional variation in dopamine dysfunction within the striatum in schizophrenia is not without precedent. In Parkinson's disease, dopamine depletion is more pronounced in the putamen and posterior regions [73], while in methamphetamine abuse de- pletion is more notable in the caudate and dorsal regions [62].

The present study has a number of limitations. The ascertainment procedures were different at the two sites, the Hong Kong sample was limited to patients requiring inpatient treatment, while the Halifax sample included patients entering care as in- or out-patients. The Hong Kong sample was nearly equally split between males and females, while the Halifax sample was only one-quarter female. The origin of the gender differences is unclear, other studies from the Hong Kong EASY program also are gender balanced [74], and studies from Halifax as well as other Vancouver, Canada are male predominant $[75,76]$. Different clinical instruments were used between sites for assessment of several key clinical features of illness. This could contribute to discrepant findings between sites, however, has the advantage of strengthening the confidence in convergent evidence between sites. Specifically, the two programs used different assessment scales for movement disorders. These may have somewhat different properties, the Simpson-Angus scale for example is highly weighted towards rigidity as a defining characteristic of Parkinsonism, and lacks an item for bradykinesia. However, the similar associations between baseline EPS, clinical symptoms and response in both cohorts suggest these findings are robust. Similar to others [16], we used relatively lenient criteria to define the presence of EPS. Negative symptoms of schizophrenia are often elevated in groups with EPS, and there is likely some overlap in definitions. However, negative symptoms were also reported to be correlated with dystonia and dyskinesia scores [17], which are unlikely to be a consequence of observational confusion. Finally, the cognitive testing results in particular are from a small number of subjects. Although a carefully matched group was available for comparison, these results remain preliminary.

The present findings could have implications for understanding the mechanism of illness in schizophrenia. Dyskinesia in chronic, never medicated patients was associated with an increased frequency of dyskinesia but not Parkinsonism in family members [77]. However, even in chronic, never treated patients, movement disorders wax and wane, and may not be a stable trait phenomena [22]. Schizotypal subjects may provide a "trait" reference point, and interestingly appear to have smaller caudate and putamen volumes [78,79]. In contrast, in most studies of first episode subjects, striatal volumes do not show statistically significant differences from healthy subjects [31,33,47-53]. Extrapyramidal symptoms and signs, as well as related cognitive dysfunction may be dimensional rather than typological features of schizophrenia. Severity may be state-dependent. The relationships between EPS and brain structures are likely to be moderated by treatment, and possibly by phase of illness as well. First episode studies, and investigation of subjects treated with medications that do not affect EPS, cognition or brain structure will allow better understanding of these illness domains in schizophrenia. 
This work was supported by the Canadian Institutes of Health Research (NET-54013), the Michael Smith Foundation for Health Research and investigator-initiated grants from Janssen-Ortho of Canada and Eli Lilly Canada. Additional funding for scanning was provided by the Queen Elizabeth-II Hospital Health Science Research Foundation and the Department of Psychiatry, Dalhousie University. Dr. Kopala was supported by a Clinical Scientist Award from Dalhousie University.

1 Torrey E F. Studies of individuals with schizophrenia never treated with antipsychotic medications: A review. Schizophr Res, 2002, 58: 101-115

2 Turner T. Rich and mad in Victorian England. Psychol Med, 1989, 19: $29-44$

3 Fenton W S. Prevalence of spontaneous dyskinesia in schizophrenia. J Clin Psychiatry, 2000, 61 (Suppl 4): 10-14

4 Rogers D. The motor disorders of severe psychiatric illness: A conflict of paradigms. Br J Psychiatry, 1985, 147: 221-232

5 Koning J P, Tenback D E, van Os J, et al. Dyskinesia and parkinsonism in antipsychotic-naive patients with schizophrenia, first-degree relatives and healthy controls: A meta-analysis. Schizophr Bull, 2010, 36: 723-731

6 Koning J P, Kahn R S, Tenback D E, et al. Movement disorders in nonpsychotic siblings of patients with nonaffective psychosis. Psychiatry Res, 2011, 188: 133-137

7 Chan R C, Gottesman I I. Neurological soft signs as candidate endophenotypes for schizophrenia: A shooting star or a Northern star? Neurosci Biobehav Rev, 2008, 32: 957-971

8 Chan R C, Xu T, Heinrichs R W, et al. Neurological soft signs in schizophrenia: A meta-analysis. Schizophr Bull, 2010, 36: 10891104

9 Peralta V, Cuesta M J, Martinez-Larrea A, et al. Differentiating primary from secondary negative symptoms in schizophrenia: A study of neuroleptic-naive patients before and after treatment. Am J Psychiatry, 2000, 157: 1461-1466

10 Puri B K, Barnes T R E, Chapman M J, et al. Spontaneous dyskinesia in first episode schizophrenia. J Neurol Neurosurg Psychiatry, 1999, 66: 76-78

11 Gervin M, Browne S, Lane A, et al. Spontaneous abnormal involuntary movements in first-episode schizophrenia and schizophreniform disorder: Baseline rate in a group of patients from an Irish catchment area. Am J Psychiatry, 1998, 155: 1202-1206

12 Kopala L C, Good K P, Fredrikson D, et al. Risperidone in first-episode schizophrenia: Improvement in symptoms and pre-existing extrapyramidal signs. Int J Psychiatry Clin Practice, 1998, 2: S19-S25

13 Fenn D S, Moussaoui D, Hoffman W F, et al. Movements in never-medicated schizophrenics: A preliminary study. Psychopharmacology, 1996, 123: 206-210

14 Chorfi M, Moussaoui D. Les schizophrènes jamais traités n'ont pas de mouvements anormaux type dyskinésie tardive. L'Encéphale, 1985, 9: 263-265

15 Caligiuri M P, Lohr J B, Jeste D V. Parkinsonism in neurolepticnaive schizophrenic patients. Am J Psychiatry, 1993, 150: 1343-1348

16 Chatterjee A, Chakos M, Koreen A, et al. Prevalence and clinical correlates of extrapyramidal signs and spontaneous dyskinesia in never-medicated schizophrenic patients. Am J Psychiatry, 1995, 152: 1724-1729

17 Honer W G, Kopala L C, Rabinowitz J. Extrapyramidal symptoms and signs in first-episode, antipsychotic exposed and non-exposed patients with schizophrenia or related psychotic illness. J Psychopharmacol, 2005, 19: 277-285

18 Peralta V, Cuesta M J. Neuromotor abnormalities in neuroleptic-naive psychotic patients: Antecedents, clinical correlates, and prediction of treatment response. Compr Psychiatry, 2011, 52: 139-145

19 Bocti C, Black D N, Waddington J L. Dyskinesia in patients with schizophrenia never treated with antipsychotics: Conceptual and pathophysiological implications. In: Bedard M A, Agid Y, Chouinard $\mathrm{S}$, et al., eds. Mental and Behavioral Dysfunction in Movement Disorders. Totawa, N.J.: Humana Press, 2003. 489-498
20 McCreadie R G, Srinivasan T N, Padmavati R, et al. Extrapyramidal symptoms in unmedicated schizophrenia. J Psychiatric Res, 2005, 39: 261-266

21 McCreadie R G, Thara R, Padmavati R, et al. Structural brain differences between never-treated patients with schizophrenia, with and without dyskinesia, and normal control subjects. Arch Gen Psychiatry, 2002, 59: 332-336

22 McCreadie R G, Padmavati R, Thara R, et al. Spontaneous dyskinesia and parkinsonism in never-medicated, chronically ill patients with schizophrenia: 18-month follow-up. Br J Psychiatry, 2002, 181: 135-137

23 McCreadie R G, Latha S, Thara R, et al. Poor memory, negative symptoms and abnormal movements in never-treated Indian patients with schizophrenia. Br J Psychiatry, 1997, 171: 360-363

24 Simpson G M, Angus J W S. A rating scale for extrapyramidal side effects. Acta Psychiatr Scand, 1970, 10: 199-208

25 Guy W. ECDEU Assessment Manual for Psychopharmacology, revised. (Publication ADM 76-338). Rockville Pike, MD: US Department of Health, Education, and Welfare, 1976

26 Chouinard G, Annable L, Ross-Chouinard A, et al. Ethopropazine and benztropine in neuroleptic-induced Parkinsonism. J Clin Psychiatry, 1979, 40: 147-152

27 Peralta V, Cuesta M J. Negative, parkinsonian, depressive and catatonic symptoms in schizophrenia: A conflict of paradigms revisited. Schizophr Res, 1999, 40: 245-253

28 Kay S, Fiszbein A, Opler L A. The positive and negative syndrome scale (PANSS) for schizophrenia. Schizophr Bull, 1987, 13: 261-276

29 Wechsler D. Wechsler Memory Scale: Revised Manual. New York: Harcourt Brace Jovanovich, 1987

30 Nelson H E. A modified card sorting test sensitive to frontal lobe defects. Cortex, 1976, 12: 313-324

31 Lang D J, Kopala L C, Vandorpe R A, et al. An MRI study of basal ganglia volumes in first-episode schizophrenia patients treated with risperidone. Am J Psychiatry, 2001, 158: 625-631

32 Lang D J, Kopala L C, Vandorpe R A, et al. Reduced basal ganglia volumes after switching to olanzapine in chronically treated patients with schizophrenia. Am J Psychiatry, 2004, 161: 1829-1836

33 Gunduz $\mathrm{H}$, Wu H, Ashtari M, et al. Basal ganglia volumes in firstepisode schizophrenia and healthy comparison subjects. Biol Psychiatry, 2002, 51: 801-808

34 Cortese L, Caligiuri M P, Malla A K, et al. Relationship of neuromotor disturbances to psychosis symptoms in first-episode neuroleptic-naive schizophrenia patients. Schizophr Res, 2005, 75: 65-75

35 Jager A D. Abnormal movements in first episode psychosis. Australas Psychiatry, 2000, 18: 38-41

36 Chakos M, Ma J, Alvir J, et al. Incidence and correlates of tardive dyskinesia in first episode of schizophrenia. Arch Gen Psychiatry, 1996, 53: 313-319

37 Schröder J, Niethammer G, Geider F-J, et al. Neurological soft signs in schizophrenia. Schizophr Res, 1992, 6: 25-30

38 Whitty P, Clarke M, Browne S, et al. Prospective evaluation of neurological soft signs in first-episode schizophrenia in relation to psychopathology: State versus trait phenomena. Psychol Med, 2003, 33: 1479-1484

39 Kopala L C, Good K P, Honer W G. Extrapyramidal signs and clinical symptoms in first-episode schizophrenia: Response to low-dose risperidone. J Clin Psychopharmacol, 1997, 17: 308-313

40 Schröder J, Silvestri S, Bubeck B, et al. D2 dopamine receptor upregulation, treatment response, neurological soft signs, and extrapyramidal side effects in schizophrenia. Biol Psychiatry, 1998, 43: 660-665

41 Waddington J L, Youssef H A. The lifetime outcome and involuntary movements of schizophrenia never treated with neuroleptic drugs. $\mathrm{Br}$ J Psychiatry, 1990, 156: 106-108

42 Pantelis C, Stuart G W, Nelson H E, et al. Spatial working memory deficits in schizophrenia: Relationship with tardive dyskinesia and negative symptoms. Am J Psychiatry, 2001, 158: 1276-1285

43 Quinn J, Meagher D, Murphy P, et al. Vulnerability to involuntary movements over a lifetime trajectory of schizophrenia approaches 
$100 \%$, in association with executive (frontal) dysfunction. Schizophr Res, 2001, 49: 79-87

44 Meyer-Lindenberg A, Miletich R S, Kohn P D, et al. Reduced prefrontal activity predicts exaggerated striatal dopaminergic function in schizophrenia. Nat Neurosci, 2002, 5: 267-271

45 McGowan S, Lawrence A D, Sales T, et al. Presynaptic dopamine dysfunction in schizophrenia. Arch Gen Psychiatry, 2004, 61: 134-142

46 Meyer-Lindenberg A, Kohn P D, Kolachana B, et al. Midbrain dopamine and prefrontal function in humans: Interaction and modulation by COMT genotype. Nat Neurosci, 2005, 8: 828-834

47 Shihabuddin L, Buchsbaum M S, Hazlett E A, et al. Dorsal striatal size, shape, and metabolic rate in never-medicated and previously medicated schizophrenics performing a verbal learning task. Arch Gen Psychiatry, 1998, 55: 235-243

48 DeLisi L E, Hoff A L, Schwartz J E, et al. Brain morphology in firstepisode schizophrenic-like psychotic patients: A quantitative magnetic resonance imaging study. Biol Psychiatry, 1991, 29: 159-175

49 Chakos M H, Lieberman J A, Bilder R M, et al. Increase in caudate nuclei volumes of first-episode schizophrenic patients taking antipsychotic drugs. Am J Psychiatry, 1994, 151: 1430-1436

50 Gur R E, Maany V, Mozley P D, et al. Subcortical MRI volumes in neuroleptic-naive and treated patients with schizophrenia. Am J Psychiatry, 1998, 155: 1711-1717

51 Glenthoj A, Glenthoj B Y, Mackeprang T, et al. Basal ganglia volumes in drug-naive first-episode schizophrenia patients before and after short-term treatment with either a typical or an atypical antipsychotic drug. Psychiatry Res, 2007, 154: 199-208

52 Crespo-Facorro B, Roiz-Santiáñez R, Pelayo-Terán J M, et al. Caudate nucleus volume and its clinical and cognitive correlations in first episode schizophrenia. Schizophr Res, 2007, 91: 87-96

53 Ballmaier M, Schlagenhauf F, Toga A W, et al. Regional patterns and clinical correlates of basal ganglia morphology in non-medicated schizophrenia. Schizophr Res, 2008, 106: 140-147

54 Keshevan M S, Rosenberg D, Sweeney J A, et al. Decreased caudate volume in neuroleptic-naive psychotic patients. Am J Psychiatry, 1998, 155: 774-778

55 Westmoreland Corson P, Nopoulos P, Miller D D, et al. Change in basal ganglia volume over 2 years in patients with schizophrenia: Typical versus atypical neuroleptics. Am J Psychiatry, 1999, 156: 1200-1204

56 Ebdrup B H, Glenthøj B, Rasmussen H, et al. Hippocampal and caudate volume reductions in antipsychotic-naive first-episode schizophrenia. J Psychiatry Neurosci, 2010, 35: 95-104

57 Rajarethinam R, Upadhyaya A, Tsou P, et al. Caudate volume in offspring of patients with schizophrenia. Br J Psychiatry, 2007, 191: 258-259

58 Eliez S, Barnea-Goraly N, Schmitt E J, et al. Increased basal ganglia volumes in velo-cardio-facial syndrome (deletion 22q11.2). Biol Psychiatry, 2002, 52: 68-70

59 Kates W R, Burnette C P, Bessette B A, et al. Frontal and caudate alterations in velocardiofacial syndrome (deletion at chromosome 22q11.2). J Child Neurol, 2004, 19: 337-342

60 Campbell L E, Daly E, Toal F, et al. Brain and behaviour in children with 22q11.2 deletion syndrome: A volumetric and voxel-based morphometry MRI study. Brain, 2006, 129: 1218-1228

61 Wilson J M, Levey A I, Bergeron C, et al. Striatal dopamine, dopa- mine transporter, and vesicular monoamine transporter in chronic cocaine users. Ann Neurol, 1996, 40: 428-439

62 Moszczynska A, Fitzmaurice P, Ang L, et al. Why is Parkinsonism not a feature of human methamphetamine users? Brain, 2004, 127: 363-370

63 Chang L, Cloak C, Patterson K, et al. Enlarged striatum in abstinent methamphetamine abusers: A possible compensatory response. Biol Psychiatry, 2005, 57: 967-974

64 Jacobsen L K, Giedd J N, Gottschalk C, et al. Quantitative morphology of the caudate and putamen in patients with cocaine dependence. Am J Psychiatry, 2001, 158: 486-489

65 Keshavan M S, Bagwell W W, Haas G L, et al. Changes in caudate volume with neuroleptic treatment. Lancet, 1994, 344: 1434

66 Chakos M H, Lieberman J A, Alvir J, et al. Caudate nuclei volumes in schizophrenic patients treated with typical antipsychotics or clozapine. Lancet, 1995, 345: 456-457

67 Scheepers F E, de Wied C C, Pol H E, et al. The effects of clozapine on caudate nucleus volume in schizophrenic patients previously treated with typical antipsychotics. Neuropsychopharmacol, 2001, 24: 47-54

68 Frazier J A, Giedd J N, Kaysen D, et al. Childhood-onset schizophrenia: Brain MRI rescan after 2 years of clozapine maintenance treatment. Am J Psychiatry, 1996, 153: 564-566

69 Hietala J, Syvalahti E, Vilkman H, et al. Depressive symptoms and presynaptic dopamine function in neuroleptic-naive schizophrenia. Schizophr Res, 1999, 35: 41-50

70 Hietala J, Syvalahti E, Vuorio K, et al. Presynaptic dopamine function in striatum of neuroleptic-naive schizophrenic patients. Lancet, 1995, 346: 1130-1131

71 Reith J, Benkelfat C, Sherwin A, et al. Elevated dopa decarboxylase activity in living brain of patients with psychosis. Proc Natl Acad Sci USA, 1994, 91: 11651-11654

72 Grunder G, Vernaleken I, Muller M J, et al. Subchronic haloperidol downregulates dopamine synthesis capacity in the brain of schizophrenic patients in vivo. Neuropsychopharmacol, 2003, 28: 787-794

73 Kish S J, Shannak K, Hornykiewicz O. Uneven pattern of dopamine loss in the striatum of patients with idiopathic Parkinson's disease. New Engl J Med, 1988, 318: 876-880

74 Chen E, Hui C, Lam M, et al. Maintenance treatment with quetiapine versus discontinuation after one year of treatment in patients with remitted first episode psychosis: Randomised controlled trial. BMJ, 2010, 341: c4024

75 Smith G N, MacEwan G W, Kopala L C, et al. Prenatal tobacco exposure and obstetrical, developmental and substance use problems in first-episode psychosis. Schizophr Res, 2010, 119: 271-272

76 Smith G N, Wong H, MacEwan G W, et al. Predictors of starting to smoke cigarettes in patients with first episode psychosis. Schizophr Res, 2009, 108: 258-264

77 McCreadie R G, Thara R, Srinivasan T N, et al. Spontaneous dyskinesia in first-degree relatives of chronically ill, never-treated people with schizophrenia. Br J Psychiatry, 2003, 183: 45-49

78 Levitt J J, McCarley R W, Dickey C C, et al. MRI study of caudate nucleus volume and its cognitive correlates in neuroleptic-naive patients with schizotypal personality disorder. Am J Psychiatry, 2002, 159: 1190-1197

79 Shihabuddin L, Buchsbaum M S, Hazlett E A, et al. Striatal size and relative glucose metabolic rate in schizotypal personality disorder and schizophrenia. Arch Gen Psychiatry, 2001, 58: 877-884

Open Access This article is distributed under the terms of the Creative Commons Attribution License which permits any use, distribution, and reproduction in any medium, provided the original author(s) and source are credited. 